\title{
THE LINEAR STABILITY OF TRAVELING WAVE SOLUTIONS FOR A REACTING FLOW MODEL WITH SOURCE TERM
}

\author{
BY \\ LING HSIAO AND RONGHUA PAN \\ Institute of Mathematics, Academia Sinica, Beijing, 100080, P.R. China
}

\begin{abstract}
In this paper, we consider a $3 \times 3$ system for a reacting flow model with a source term in [7]. This model can be considered as a relaxation approximation to $2 \times 2$ systems of conservation laws, which include the well-known $p$-system. From this viewpoint, by introducing the new waves through time-asymptotic expansion and using the $L^{2}$ energy method, we establish the global existence and the linear stability of traveling wave solutions.
\end{abstract}

1. Introduction. Consider the following system:

$$
\begin{aligned}
(\rho r)_{t}+(\rho u r)_{x} & =S, \\
(\rho s)_{t}+(\rho u s)_{x} & =-S, \\
(\rho u)_{t}+\left(p+\rho u^{2}\right)_{x} & =0,
\end{aligned}
$$

which was proposed by R. J. LeVeque and others in [7] to model the motion of reacting gas with two modes. Here, $\rho r$ is the density of the major mode and $\rho s$ corresponds to the minor mode, $r+s=1$. $u$ is the velocity, and $p=\rho c^{2}(r+\beta s)$ is the pressure, which can be derived by Avogadro's Law. Here, $c$ is the sound speed of the major mode. The parameter $\beta$ provides some tenuous link with real physics, if it is considered as the number of molecules of the minor species produced from one molecule of the major

Received August 7, 1997.

2000 Mathematics Subject Classification. Primary 35L60, 35F25.

Key words and phrases. Linear stability, traveling wave, relaxation approximation, diffusive waves, energy estimates, reacting flow.

The first author was supported partially by NSF of China.

Current address of second author: S.I.S.S.A. Via Beirut, N. 2-4, 34014, Trieste, Italy.

E-mail address: panrh@sissa.it 
species. $S$ is the source term

$$
\begin{aligned}
S & =\frac{\rho\left(r_{E}(\rho)-r\right)}{\tau} \\
& =-\frac{\rho\left(s_{E}(\rho)-s\right)}{\tau}
\end{aligned}
$$

where $\tau$ is a reaction time, and $r_{E}(\rho)$ and $s_{E}(\rho)$ are equilibrium distributions. The reader is referred to [7] for more physical and numerical background.

The Lagrangian form of (1.1) is

$$
\begin{aligned}
v_{t}-u_{x} & =0 \\
u_{t}+p_{x} & =0 \\
p_{t}+\frac{p}{v} v_{t} & =\frac{p_{E}(v)-p}{\tau}
\end{aligned}
$$

where $v$ is specific volume, $r_{1 E}=c^{2}\left(r_{E}+\beta\left(1-r_{E}\right)\right)$, and $p_{E}(v)=\frac{r_{1 E}(v)}{v}$. On the other hand, (1.3) can be considered as a relaxation approximation of the following conservation laws $($ as $\tau \rightarrow 0)$ :

$$
\begin{aligned}
v_{t}-u_{x} & =0, \\
u_{t}+p_{E}(v)_{x} & =0 .
\end{aligned}
$$

Consider the Riemann problem of (1.4) with the following data:

$$
(v(x, 0), u(x, 0))=\left(v_{0}(x), u_{0}(x)\right),
$$

where

$$
\left(v_{0}(x), u_{0}(x)\right)= \begin{cases}\left(v_{-}, u_{-}\right), & x<0 \\ \left(v_{+}, u_{+}\right), & x>0\end{cases}
$$

and $\left(v_{-}, u_{-}\right)$and $\left(v_{+}, u_{+}\right)$are two constant states.

We give the following hypotheses: for some constants $c_{1}$ and $d_{1}$ such that $0<c_{1}<$ $v_{-}, v_{+}<d_{1}<+\infty$, it follows for $v \in\left[c_{1}, d_{1}\right]$ and for some positive constants $a_{1}$ and $a_{2}$ that

$\left.H_{1}\right) p_{E}^{\prime}(v)<-a_{1}<0$

$\left.H_{2}\right) p_{E}^{\prime \prime}(v)>a_{2}>0$,

$\left.H_{3}\right)\left|p_{E}^{\prime}(v)\right|<\min \left\{\frac{p_{+}}{v_{+}}, \frac{p_{-}}{v_{-}}\right\}$,

$\left.H_{4}\right) p_{E}(v), p_{E}^{\prime}, p_{E}^{\prime \prime}, p_{E}^{\prime \prime \prime}$ are bounded, with $p_{\mp}=p_{E}\left(v_{\mp}\right)$. Here $H_{3}$ ) relates to the so-called sub-characteristic condition (see [8]).

Under $\left.\left.H_{1}\right)-H_{2}\right),(1.4)$ is strictly hyperbolic and genuinely nonlinear, with eigenvalues

$$
\lambda_{1}=-\left(-p_{E}^{\prime}(v)\right)^{\frac{1}{2}}<0<\left(-p_{E}^{\prime}(v)\right)^{\frac{1}{2}}=\lambda_{2}
$$

Definition 1. A discontinuity $\left(\sigma ; v_{-}, u_{-} ; v_{+}, u_{+}\right)$of weak solutions for $(2.1)$ is called a shock wave satisfying the entropy condition if 
A. The Rankine-Hugoniot Condition is satisfied and the speed $\sigma\left(v_{+}, v_{-}\right)$is defined, namely,

$$
\begin{aligned}
\sigma\left(v_{+}-v_{-}\right) & =-\left(u_{+}-u_{-}\right), \\
\sigma\left(u_{+}-u_{-}\right) & =p_{E}\left(v_{+}\right)-p_{E}\left(v_{-}\right), \\
\sigma\left(v_{+}, v_{-}\right) & =\mp\left[-\frac{p_{E}\left(v_{+}\right)-p_{E}\left(v_{-}\right)}{v_{+}-v_{-}}\right]^{\frac{1}{2}} ;
\end{aligned}
$$

B. The entropy condition holds, namely for any $v$ between $v_{-}$and $v_{+}$,

$$
\begin{array}{ll}
\sigma^{2}\left(v, v_{+}\right)<\sigma^{2}\left(v_{-}, v_{+}\right)<\sigma^{2}\left(v, v_{-}\right), & \text {for } \sigma>0, \\
\sigma^{2}\left(v, v_{+}\right)>\sigma^{2}\left(v_{-}, v_{+}\right)>\sigma^{2}\left(v, v_{-}\right), & \text {for } \sigma<0,
\end{array}
$$

where $\sigma^{2}\left(v, v^{*}\right)=-\frac{p_{E}(v)-p_{E}\left(v^{*}\right)}{v_{-} v^{*}}$, and $v^{*}=v_{-}$or $v_{+}$.

For any given single weak shock wave $\left(\sigma ; v_{-}, u_{-} ; v_{+}, u_{+}\right)$of $(1.4)$ satisfying the entropy conditions, it has been proved in [13] that (1.3) admits a smooth traveling wave solution:

$$
(\bar{v}, \bar{u}, \bar{p})(x, t)=(\bar{v}, \bar{u}, \bar{p})(\xi)
$$

with

$$
\bar{v}( \pm \infty)=v_{ \pm}, \quad \bar{u}( \pm \infty)=u_{ \pm}, \quad \bar{p}( \pm \infty)=p_{ \pm}
$$

where $\xi=\frac{x-\sigma t}{\tau}$. The nonlinear stability of $(\bar{v}, \bar{u}, \bar{p})$ for (1.3) has been established by Pan in [13] under the following zero excess mass restriction

$$
\int_{-\infty}^{+\infty}\left(v_{0}(x)-\bar{v}(x, 0)\right) d x=0, \quad \int_{-\infty}^{+\infty}\left(u_{0}(x)-\bar{u}(x, 0)\right) d x=0
$$

which says that this kind of perturbation on a shock profile produces only a translation. A similar result can be found in [15].

For $2 \times 2$ relaxation models, the stability of elementary waves has been obtained in [8], where the corresponding equilibrium equation is a scalar conservation law. Therefore, a generic perturbation of a shock profile indeed produces only a translation. However, as mentioned in [9], [11], and [14], since the equilibrium system for (1.3), i.e., (1.4) is a $2 \times 2$ system, a generic perturbation of a single shock front will create not only a translation but also some new waves. By this observation, the stability of a single shock front for a linearized system of the following rate-type viscoelastic system

$$
\begin{aligned}
v_{t}-u_{x} & =0 \\
u_{t}+p_{x} & =0 \\
{[p+E v]_{t} } & =\frac{p_{R}(v)-p}{\tau},
\end{aligned}
$$

is proved in [11] without the restriction (1.8). For the system (1.9), the nonlinear stability of two-mode shock waves has been shown in [5], where a generic perturbation indeed produces only the translations of 1- and 2-shock profiles. For this system, the nonlinear stability of a single shock under (1.8) and the nonlinear stability of rarefaction waves have been established in [3] and [4] respectively.

It is known that the second order of the Chapman-Enskog-type expansion of (1.3) is a $2 \times 2$ viscous conservation law. Based on this fact, we construct a diffusive wave 
that carries the "excess mass" along the right eigenvector belonging to the other family of the shock front. We also introduce a high-order correction that carries the zero net "mass". Then we can establish the linear stability result similar to [11] as the first step of nonlinear stability.

It should be noticed that the principle part of (1.9) is linear, but the principle part of (1.3) is nonlinear; thus, more difficulties occur certainly. It should also be pointed out that, to show the linear stability result with the system (1.9), one only needs to deal with a linear system (see [11]); however, we have to work with a nonlinear system instead.

Now, let us turn to our main result of this paper.

Since we are only interested in the large-time behavior of the solution to (1.3) for fixed $\tau$, we can assume that $\tau=1$ without loss of generality. Assume that $\left(v_{-}, u_{-}\right)$and $\left(v_{+}, u_{+}\right)$can be connected by a 2 -shock of (1.4) with speed $\sigma>0$. Then, $v_{-}<v_{+}$. The case for $\sigma<0$ can be treated similarly. Then the linearized system of (1.3) at the shock profile $(\bar{v}, \bar{u}, \bar{p})$ is

$$
\begin{aligned}
v_{t}-u_{x} & =0, \\
u_{t}+p_{x} & =0 \\
p_{t}+\frac{p}{v} v_{t} & =p_{E}(v)-p, \\
p_{E}(v) & =p_{E}(\bar{v})+p_{E}^{\prime}(\bar{v})(v-\bar{v}) .
\end{aligned}
$$

In the following we will consider (1.10) with the initial data:

$$
(v(x, 0), u(x, 0), p(x, 0))=\left(v_{0}(x), u_{0}(x), p_{0}(x)\right),
$$

which is a generic perturbation of the shock profile $(\bar{v}, \bar{u}, \bar{p})$ in the following sense:

$$
\int_{-\infty}^{+\infty}\left(v_{0}(x)-\bar{v}(x), u_{0}(x)-\bar{u}(x)\right) d x=\left(\delta_{1}, \delta_{2}\right)
$$

for suitable small numbers $\delta_{1}, \delta_{2}$.

Suppose that the shock wave is sufficiently weak, i.e.,

$$
\eta \equiv\left|v_{+}-v_{-}\right|<<1
$$

so that the vectors $\left(v_{+}-v_{-}, u_{+}-u_{-}\right)^{t}$ and $\left(1, \sqrt{-p_{E}^{\prime}\left(v_{-}\right)}\right)^{t}$ are linearly independent (the vector $\left(1, \sqrt{-p_{E}^{\prime}\left(v_{-}\right)}\right)^{t}$ is the right eigenvector belonging to the 1-family for (1.4)). Then there are constants $x_{0}$ and $\delta$, determined uniquely, such that

$$
\begin{aligned}
& \int_{-\infty}^{+\infty}\left(v_{0}(x)-\bar{v}(x), u_{0}(x)-\bar{u}(x)\right) d x \\
& \quad=x_{0}\left(v_{+}-v_{-}, u_{+}-u_{-}\right)+\delta\left(1, \sqrt{-p_{E}^{\prime}\left(v_{-}\right)}\right) .
\end{aligned}
$$

We note that the first part of the right-hand side of (1.14) produces a translation of the shock profile (see (3.11) below for instance) while the other part will create some new waves that will be determined later in Sec. 3 .

The main result of the present paper is the following stability theorem.

Theorem 1. Suppose that $\left.\left.H_{1}\right)-H_{4}\right)$ hold. Let $(\bar{v}, \bar{u}, \bar{p})\left(x+x_{0}-\sigma t\right)$ be the shock profile of (1.3) corresponding to the shock wave $\left(\sigma ; v_{-}, u_{-} ; v_{+}, u_{+}\right)$of $(1.4)$, and let $x_{0}$ be the 
translation uniquely determined by (1.14). Suppose that the initial data $\left(v_{0}, u_{0}, p_{0}\right)(x)$ satisfies

$$
\begin{aligned}
& \left(\left(v_{0}, u_{0}, p_{0}\right)(x)-(\bar{v}, \bar{u}, \bar{p})\left(x+x_{0}\right)\right) \in H^{2}(\mathbf{R}), \\
& \int_{-\infty}^{+\infty}\left(1+x^{2}\right)\left(v_{0}(x)-\bar{v}\left(x+x_{0}, 0\right)\right)^{2} d x<\delta_{3}, \\
& \int_{-\infty}^{+\infty}\left(1+x^{2}\right)\left(u_{0}(x)-\bar{u}\left(x+x_{0}, 0\right)\right)^{2} d x<\delta_{4},
\end{aligned}
$$

for some positive constants $\delta_{3}, \delta_{4}$. Then there exist positive constants $\delta_{0}$ and $\varepsilon_{0}$, such that if $\delta<\delta_{0}, \eta=\left|v_{+}-v_{-}\right|<\delta_{0}$, and

$$
\left.\|\left(\left(v_{0}, u_{0}, p_{0}\right)(x)-(\bar{v}, \bar{u}, \bar{p})\left(x+x_{0}, 0\right)\right)\right) \|_{H^{2}}^{2}+\delta_{3}+\delta_{4} \leq \varepsilon_{0},
$$

then there exists a unique smooth global solution $U \equiv(v, u, p)$ to $(1.10)-(1.11)$ satisfying

$$
\begin{gathered}
U(x, t)-\bar{U}\left(x+x_{0}-\sigma t\right) \in C\left([0,+\infty) ; H^{2}(\mathbf{R})\right), \\
\lim _{t \rightarrow+\infty}\left\|U(x, t)-\bar{U}\left(x+x_{0}-\sigma t\right)\right\|_{H^{1}}=0,
\end{gathered}
$$

where $\bar{U} \equiv(\bar{v}, \bar{u}, \bar{p})$. Hereafter we use the following notation for simplicity:

$$
\left\|\left(f_{1}, f_{2}, \ldots, f_{l}\right)\right\|_{H^{m}}^{2} \equiv \sum_{i=1}^{l}\left\|f_{i}\right\|_{H^{m}}^{2},
$$

with $l \geq 1, m \geq 0$, and $H^{0}=L^{2}$.

We will end this introduction by outlining the plan of the remaining part of this paper. In Sec. 2, the traveling wave solutions are discussed carefully. The diffusive waves and the high-order correction are studied in Sec. 3. Based on the results in Secs. 2-3, we use time-asymptotic expansion and energy methods to prove the linear stability in Sec. 4 .

2. Traveling wave solutions. A traveling wave solution of the reacting flow system

$$
\begin{aligned}
v_{t}-u_{x} & =0, \\
u_{t}+p_{x} & =0, \\
p_{t}+\frac{p}{v} v_{t} & =p_{E}(v)-p,
\end{aligned}
$$

corresponding to the 2 -shock $\left(\sigma ; v_{-}, u_{-} ; v_{+}, u_{+}\right)$of $(1.4)$ is the solution of the form

$$
(v, u, p)(x, t)=(\bar{v}, \bar{u}, \bar{p})(\xi), \quad \xi=x-\sigma t
$$

satisfying

$$
(\bar{v}, \bar{u}, \bar{p})(\mp \infty)=\left(v_{\mp}, u_{\mp}, p_{\mp}\right) .
$$

Under $\left.H_{1}\right)-H_{3}$ ), it is proved in [13] that (2.1) admits a smooth traveling wave solution, which is unique up to a shift of $\xi$, provided that $\left|v_{+}-v_{-}\right|$is small. We now sketch the proof and give some properties of the traveling wave solution. 
It is easy to know that $(\bar{v}, \bar{u}, \bar{p})$ satisfies

$$
\begin{aligned}
-\sigma \overline{v_{\xi}}-\overline{u_{\xi}} & =0, \\
-\sigma \overline{u_{\xi}}+\overline{p_{\xi}} & =0, \\
\sigma\left(\sigma^{2}-\frac{\bar{p}}{\bar{v}}\right) \bar{v}_{\xi} & =p_{E}(\bar{v})-\bar{p} .
\end{aligned}
$$

Thus, we have

$$
\begin{aligned}
& \bar{u}=u_{-}-\sigma\left(\bar{v}-v_{-}\right), \\
& \bar{p}=p_{-}-\sigma^{2}\left(\bar{v}-v_{-}\right)=p_{+}+\sigma^{2}\left(v_{+}-\bar{v}\right) .
\end{aligned}
$$

It is not difficult to get from (2.4) that

$$
\bar{v}_{\xi}=g(\bar{v})
$$

where

$$
g(\bar{v})=-\frac{\left(p_{E}(\bar{v})-p_{-}\right)+\sigma^{2}\left(\bar{v}-v_{-}\right)}{\sigma\left(\frac{\bar{p}}{v}-\sigma^{2}\right)} .
$$

Due to entropy and sub-characteristic conditions (see [8])

$$
\sigma^{2}<\frac{\bar{p}}{\bar{v}}
$$

(which can be shown by $H_{3}$ and the smallness of $\left|v_{+}-v_{-}\right|$via an implicit function theorem argument), we see that $v_{-}$and $v_{+}$are the only roots of $g(\bar{v})=0$ and

$$
\bar{v}_{\xi}>0 \text { for } \bar{v} \in\left(v_{-}, v_{+}\right) \text {. }
$$

So, $\int_{v_{1}}^{\bar{v}} \frac{d \eta}{g(\eta)}$ is finite and monotone with respect to $\bar{v}$ for any given $\bar{v}, v_{1} \in\left(v_{-}, v_{+}\right)$.

It can be shown that

$$
\begin{aligned}
& g(v)=O\left(\left|v-v_{+}\right|\right) \quad \text { as } v \rightarrow v_{+} \\
& g(v)=O\left(\left|v-v_{-}\right|\right) \quad \text { as } v \rightarrow v_{-}
\end{aligned}
$$

These imply

$$
\int_{v_{1}}^{v_{+}} \frac{d \eta}{g(\eta)}=+\infty, \quad \int_{v_{1}}^{v_{-}} \frac{d \eta}{g(\eta)}=-\infty, \quad \forall v_{1} \in\left(v_{-}, v_{+}\right) .
$$

Thus, by integrating (2.5), we obtain

$$
\xi=\int_{v_{1}}^{\bar{v}(\xi)} \frac{d \eta}{g(\eta)}
$$

This gives an implicit formula for $\bar{v}(\xi)$ that is uniquely determined (up to the choice of $v_{1}$ ) due to the properties of $g$. To be definite, we take

$$
v_{1}=\frac{1}{2}\left(v_{-}+v_{+}\right)
$$

Then $\bar{u}(\xi)$ and $\bar{p}(\xi)$ can easily be determined. Therefore, we get

TheOREM 2.1. Under $\left.\left.H_{1}\right)-H_{3}\right)$, if $\left|v_{+}-v_{-}\right|$is small, (2.1) has a smooth traveling wave solution that is unique up to a shift in $\xi$ and satisfies $\bar{v}_{\xi}>0,\left(\frac{\bar{p}}{\bar{v}}\right)_{\xi}<0$, and $\inf _{\bar{v} \in\left[v_{-}, v_{+}\right] \frac{\bar{p}}{\bar{v}}=}$ $\frac{p_{+}}{v_{+}}$.

It is also easy to show that 
LEMMA 2.2.

$$
\left|\bar{v}_{\xi}\right| \leq C\left|v_{+}-v_{-}\right|, \quad\left|\bar{u}_{\xi}\right| \leq C\left|v_{+}-v_{-}\right|, \quad\left|\bar{p}_{\xi}\right| \leq C\left|v_{+}-v_{-}\right|,
$$

and it is also true for the second and the third derivatives of $\bar{v}, \bar{u}$, and $\bar{p}$, respectively.

We need the following sharper estimate, which plays a key role in our stability analysis. LEMMA 2.3.

$$
\left|\bar{v}_{\xi}\right|+\left|\bar{v}_{\xi \xi}\right|+\left|\bar{v}_{\xi \xi \xi}\right| \leq C_{1}\left(\left|v_{+}-v_{-}\right|\right) \exp \left(-C_{2}|\xi|\right),
$$

where $C_{i}(i=1,2)$ is a positive constant.

Proof. From (2.5),

$$
\begin{aligned}
\bar{v}_{\xi \xi} & =g(\bar{v})_{\xi} \\
& =-\frac{f\left(\bar{v}, \bar{v}_{\xi}\right) \bar{v}_{\xi}}{\sigma\left(\frac{\bar{p}}{v}-\sigma^{2}\right)}
\end{aligned}
$$

where

$$
f=\sigma^{2}+p_{E}^{\prime}(\bar{v})-\sigma\left(\frac{p_{+}+\sigma^{2} v_{+}}{\bar{v}^{2}}\right) \bar{v}_{\xi} .
$$

Due to the convexity of $p_{E}$, the entropy condition implies the Lax shock condition (see [1]), namely,

$$
-p_{E}^{\prime}\left(v_{+}\right)<\sigma^{2}<-p_{E}^{\prime}\left(v_{-}\right)
$$

Then we know that

$$
f(-\infty)<0 \text { and } f(+\infty)>0 .
$$

By a mean-value-theorem argument, one can prove that (cf. [13])

$$
\left|\bar{v}_{\xi \xi}\right| \leq C_{3}\left|v_{+}-v_{-}\right| \bar{v}_{\xi}
$$

for a positive constant $C_{3}$. Thus we have

$$
\begin{aligned}
f_{\xi} & =p_{E}^{\prime \prime}(\bar{v}) \bar{v}_{\xi}-\sigma\left(\frac{p_{+}+\sigma^{2} v_{+}}{\bar{v}^{2}}\right) \bar{v}_{\xi \xi}+2 \sigma\left(\frac{p_{+}+\sigma^{2} v_{+}}{\bar{v}^{3}}\right) \bar{v}_{\xi}^{2} \\
& \geq\left(p_{E}^{\prime \prime}(\bar{v})-C_{4}\left|v_{+}-v_{-}\right|\right) \bar{v}_{\xi} \\
& >0
\end{aligned}
$$

which implies that $f(\xi)$ is a strictly increasing function with respect to $\xi$, and there exists a unique $\xi_{0}$ (we can assume that $\xi_{0}=0$ without loss of generality) such that $f\left(\xi_{0}\right)=0$. This shows that

$$
\begin{array}{ll}
f(\xi)<0 & \text { for } \xi<0 \\
f(\xi)>0 & \text { for } \xi>0
\end{array}
$$

$(2.8)-(2.10)$ yield that

$$
\bar{v}_{\xi}(\xi)=\bar{v}_{\xi}(0) \exp \left(-\int_{0}^{\xi} \frac{f(\xi)}{\sigma\left(\frac{\bar{p}}{v}-\sigma^{2}\right)} d \xi\right) .
$$

Combining (2.8)-(2.11), we complete the proof of Lemma 2.3. 
3. Diffusive waves and high-order corrections. Consider

$$
\begin{aligned}
v_{t}-u_{x} & =0 \\
u_{t}+p_{x} & =0 \\
p_{t}+\frac{p}{v} v_{t} & =p_{E}(v)-p,
\end{aligned}
$$

with

$$
p_{E}(v)=p_{E}(\bar{v})+p_{E}^{\prime}(\bar{v})(v-\bar{v})
$$

where $(\bar{v}, \bar{u}, \bar{p})$ is the shock profile of (1.3) determined in Sec. 2 with a shift $x_{0}$ as in (1.14).

As mentioned in Sec. 1, a generic perturbation of a 2-shock front produces not only the translation but also some new waves along the direction of the 1-right eigenvector to carry the "excess mass" (see (1.14)). We also note that the second-order expansion of (3.1) (similar to the Chapman-Enskog expansion for the Boltzmann equation (see [8])) is the following viscous conservation laws:

$$
\begin{aligned}
v_{t}-u_{x} & =0 \\
u_{t}+p_{E}(v)_{x} & =\left[\left(p_{E}^{\prime}(v)+\frac{p_{E}(v)}{v}\right) u_{x}\right]_{x} .
\end{aligned}
$$

Motivated by the idea of [9], we construct the diffusive wave $n(x, t)\left(1, \sqrt{-p_{E}^{\prime}\left(v_{-}\right)}\right)^{t}$ with the base state $\left(v_{-}, u_{-}\right)$, which carries the "excess mass" $\delta\left(1, \sqrt{-p_{E}^{\prime}\left(v_{-}\right)}\right)^{t}$. Here $n(x, t)$ is a self-similar solution of the following equation:

$$
n_{t}+\lambda_{1}\left(v_{-}\right) n_{x}=\frac{1}{2}\left(\frac{p_{-}}{v_{-}}+p_{E}^{\prime}\left(v_{-}\right)\right) n_{x x} .
$$

It is known that (see [9], [11], and [14]), we may choose $n(x, t)$ as

$$
n(x, t) \equiv(t+1)^{-\frac{1}{2}} m(z),
$$

with $m(z)=\frac{\delta}{\sqrt{2 \pi d}} \exp \left(-\frac{z^{2}}{2 d}\right), z \equiv \frac{x-\lambda_{1}\left(v_{-}\right)(t+1)}{\sqrt{(t+1)}}$, and $d \equiv \frac{p_{-}}{v_{-}}+p_{E}^{\prime}\left(v_{-}\right)$. It is easy to see that

$$
\int_{-\infty}^{+\infty} n(x, t) d x=\delta
$$

Since (3.1) cannot be solved by the linear superposition of a translated shock profile and a diffusive wave exactly, we need some high-order corrections that carry the zero net mass. Now we perform the following time-asymptotic expansion for the solution $(v, u, p)$ :

$$
\begin{aligned}
& v=\bar{v}(y)+a(t+1)^{-\frac{1}{2}} m(z)+a_{1}(t+1)^{-1} m_{1}(z)+\cdots, \\
& u=\bar{u}(y)+b(t+1)^{-\frac{1}{2}} m(z)+b_{1}(t+1)^{-1} m_{1}(z)+\cdots, \\
& p=\bar{p}(y)+c(t+1)^{-\frac{1}{2}} m(z)+c_{1}(t+1)^{-1} m_{1}(z)+\cdots,
\end{aligned}
$$


with $y=x+x_{0}-\sigma t$. Based on (3.6), we decompose the solution as follows:

$$
\begin{aligned}
& v=\bar{v}(y)+a(t+1)^{-\frac{1}{2}} m(z)+a_{1}(t+1)^{-1} m_{1}(z)+v^{*} \\
& u=\bar{u}(y)+b(t+1)^{-\frac{1}{2}} m(z)+b_{1}(t+1)^{-1} m_{1}(z)+u^{*} \\
& p=\bar{p}(y)+c(t+1)^{-\frac{1}{2}} m(z)+c_{1}(t+1)^{-1} m_{1}(z)+p^{*}
\end{aligned}
$$

Rewrite the system (3.1) into

$$
\begin{aligned}
v_{t}-u_{x} & =0 \\
u_{t}+p_{x} & =0 \\
p_{t}+\frac{p_{-}}{v_{-}} v_{t} & =p_{E}(v)-p-F_{1} v_{t}-F_{2} v_{t},
\end{aligned}
$$

where $F_{1}=\left(\frac{p}{v}-\frac{\bar{p}}{v}\right)$ and $F_{2}=-\left(\frac{p_{-}}{v_{-}}-\frac{\bar{p}}{v}\right)$. Then we insert (3.7) into (3.8) with

$$
\begin{array}{r}
a=1, \quad b=-\lambda_{1}\left(v_{-}\right) \equiv-\lambda_{1}, \quad c=p_{E}^{\prime}\left(v_{-}\right)=-\lambda_{1}^{2} \\
a_{1}=1, \quad b_{1}=-\frac{1}{2} \lambda_{1}, \quad c_{1}=0, \quad m_{1}=-\lambda_{1}^{-1} d m_{z} .
\end{array}
$$

In a similar manner as used in [11], we get the following system for the remainder $\left(v^{*}, u^{*}, p^{*}\right)$ :

$$
\begin{aligned}
v_{t}^{*}-u_{x}^{*} & =F_{3}, \\
u_{t}^{*}+p_{x}^{*} & =F_{4}, \\
p_{t}^{*}+\frac{p_{-}}{v_{-}} v_{t}^{*} & =-p^{*}+p_{E}^{\prime}(\bar{v}) v^{*}+G_{1}+F_{5}+\sum_{i=1}^{7} I_{i},
\end{aligned}
$$

where

$$
\begin{aligned}
& F_{3}=\frac{1}{2} d \lambda_{1}^{-1}(t+1)^{-1}(z m)_{x x} \\
& F_{4}=-\frac{1}{4} d(t+1)^{-1}(z m)_{x x} \\
& F_{5}=\frac{1}{2}(t+1)^{-1}\left[(z m)_{x}+d \frac{p_{-}}{v_{-}} \lambda_{1}^{-1}(z m)_{x x}\right] \\
& G_{1}=(t+1)^{-\frac{1}{2}}\left(p_{E}^{\prime}(\bar{v})-p_{E}^{\prime}\left(v_{-}\right)\right)\left(m+d \lambda_{1}^{-1} m_{x}\right) \\
& I_{1}=-F_{1}\left[\frac{1}{2}(t+1)^{-1}(z m)_{x}+\lambda_{1}(t+1)^{-\frac{1}{2}} m_{x}\right] \\
& I_{2}=d \lambda_{1}^{-1} F_{1}\left[(t+1)^{-\frac{3}{2}}\left(m_{x}\right)+(t+1)^{-1} z m_{x x}+\lambda_{1}(t+1)^{-\frac{1}{2}} m_{x x}\right] \\
& I_{3}=-F_{2}\left[\frac{1}{2}(t+1)^{-1}(z m)_{x}+\lambda_{1}(t+1)^{-\frac{1}{2}} m_{x}\right] \\
& I_{4}=d \lambda_{1}^{-1} F_{2}\left[(t+1)^{-\frac{3}{2}}\left(m_{x}\right)+(t+1)^{-1} z m_{x x}+\lambda_{1}(t+1)^{-\frac{1}{2}} m_{x x}\right] \\
& I_{5}=-F_{1} \bar{v}_{t} \\
& I_{6}=-F_{1} v_{t}^{*} \\
& I_{7}=-F_{2} v_{t}^{*}
\end{aligned}
$$


Due to (3.9), we know that the high-order correction (the third terms in (3.7)) indeed carries the zero net mass. Then from (1.14), (3.5), and the following fact

$$
\begin{aligned}
& \int_{-\infty}^{+\infty}\left(\bar{v}\left(x+x_{0}\right)-\bar{v}(x)\right) d x=x_{0}\left(v_{+}-v_{-}\right) \\
& \int_{-\infty}^{+\infty}\left(\bar{u}\left(x+x_{0}\right)-\bar{u}(x)\right) d x=x_{0}\left(u_{+}-u_{-}\right)
\end{aligned}
$$

one has

$$
\int_{-\infty}^{+\infty} v^{*}(x, 0) d x=0, \quad \int_{-\infty}^{+\infty} u^{*}(x, 0) d x=0 .
$$

Hence (3.10) leads to

$$
\int_{-\infty}^{+\infty} v^{*}(x, t) d x=0, \quad \int_{-\infty}^{+\infty} u^{*}(x, t) d^{l} x=0 .
$$

4. Stability analysis. With the help of the results in Secs. $2-3$, we are going to prove Theorem 1 in this section.

Let us introduce

$$
\phi(x, t)=\int_{-\infty}^{x} v^{*}(y, t) d y, \quad \psi(x, t)=\int_{-\infty}^{x} u^{*}(y, t) d y, \quad w=p^{*} .
$$

In view of $(3.12)$, it is easy to see that

$$
\phi(\mp \infty)=\psi(\mp \infty)=w(\mp \infty)=0 .
$$

From (3.10), we have

$$
\begin{aligned}
\phi_{t}-\psi_{x} & =F_{6} \\
\psi_{t}+w & =F_{7} \\
w_{t}+K \phi_{x t}+w-p_{E}^{\prime}(\bar{v}) \phi_{x} & =G_{1}+F_{5}+\sum_{i=1}^{7} I_{i}
\end{aligned}
$$

with

$$
\begin{gathered}
K=\frac{p_{-}}{v_{-}} \\
F_{6}=\frac{1}{2} d \lambda_{1}^{-1}(t+1)^{-1}(z m)_{x},
\end{gathered}
$$

and

$$
F_{7}=-\frac{1}{4} d(t+1)^{-1}(z m)_{x}
$$

The weighted Poincaré inequality (see [2], Thm. 328) gives

$$
\begin{aligned}
& \left.\left|\int_{0}^{+\infty}\right| \int_{y}^{+\infty}\left(v_{0}(x)-\bar{v}\left(x+x_{0}, 0\right)-m(x, 0)-m_{1}(x, 0)\right) d x\right|^{2} d y \mid \\
& \quad \leq 4 \int_{0}^{+\infty}\left(v_{0}(x)-\bar{v}\left(x+x_{0}, 0\right)-m(x, 0)-m_{1}(x, 0)\right)^{2} x^{2} d x
\end{aligned}
$$


which implies that $\phi(x, 0)=\phi_{0}(x) \in H^{3}$ and $\left\|\phi_{0}(x)\right\|_{H^{3}}^{2} \leq C\left(\varepsilon_{0}+\delta_{0}\right)$. Similarly, $\psi(x, 0)=\psi_{0}(x) \in H^{3}$ and $\left\|\psi_{0}(x)\right\|_{H^{3}}^{2} \leq C\left(\varepsilon_{0}+\delta_{0}\right)$. In what follows, we will use $C$ to denote the generic constant independent of $t$.

We denote

$$
\begin{aligned}
L_{1} & \equiv \phi_{t}-\psi_{x}=F_{6}, \\
L_{2} & \equiv \psi_{t t}-K \psi_{x x}+\psi_{t}-A \phi_{x} \\
& =-G_{1}-F_{5}+F_{8}-\sum_{i=1}^{7} I_{i} \equiv R(x, t),
\end{aligned}
$$

with

$$
\begin{gathered}
A=-p_{E}^{\prime}(\bar{v}), \\
F_{8}=K F_{6 x}+F_{7}+F_{7 t} .
\end{gathered}
$$

It is not difficult to know that (4.4)-(4.5) give a closed system for $(\phi, \psi)$. We consider (4.4)-(4.5) with initial data:

$$
\begin{gathered}
\phi(x, 0)=\phi_{0}(x)=\int_{-\infty}^{x} v_{0}^{*}(y) d y \in H^{3}, \\
\psi(x, 0)=\psi_{0}(x)=\int_{-\infty}^{x} u_{0}^{*}(y) d y \in H^{3}, \\
\psi_{t}(x, 0)=\psi_{1}(x)=-w_{0}(x)=p_{0}^{*}(x) \in H^{2} .
\end{gathered}
$$

Due to the expansion (3.7), in view of (4.1) and (4.3), we only need to prove the following convergence result to show Theorem 1 .

ThEOREm 4.1. Suppose that the conditions in Theorem 1 hold. Then there exist positive constants $\delta_{0}$ and $\varepsilon_{0}$ such that if $\delta<\delta_{0}, \eta<\delta_{0}$, and

$$
\left\|\left(\phi_{0}, \psi_{0}\right)\right\|_{H^{3}}+\left\|\psi_{1}\right\|_{H^{2}} \leq \varepsilon_{0},
$$

then the problem (4.4)-(4.5) and (4.8) has a unique global smooth solution such that

$$
\lim _{t \rightarrow+\infty}\left(\left\|\left(\phi_{x}, \psi_{x}\right)\right\|_{H^{2}}+\left\|\psi_{t}\right\|_{H^{2}}\right)=0
$$

and

$$
\lim _{t \rightarrow+\infty} \sup _{x \in \mathbf{R}}|(\phi, \psi)(t)|=0 .
$$

We will solve the Cauchy problem (4.4)-(4.5) and (4.8) in the space

$$
X(0, T)=\left\{(\phi, \psi) \in C^{0}\left(0, T ; H^{3}\right), \psi_{t} \in C^{0}\left(0, T ; H^{2}\right)\right\}
$$

with the norm

$$
N^{2}(t)=\sup _{0 \leq \tau \leq t}\left(\|(\phi, \psi)(\tau)\|_{H^{3}}^{2}+\left\|\psi_{t}(\tau)\right\|_{H^{2}}^{2}\right) .
$$

To prove Theorem 4.1, we need the following a priori estimates. In the following, we always assume a priori that $(\phi, \psi) \in X(0, T)$ is the smooth solution of (4.4)-(4.5) and (4.8) for some $T>0$. Furthermore, we will use the fourth derivatives of $\phi$ or $\psi$ formally. 
This will not cause any trouble, since we may assume $(\phi, \psi) \in H^{4}$ first and use Fridrich's mollifier then to deal with the original case.

Lemma 4.2. Suppose the conditions in Theorem 1 are satisfied, $\delta<\delta_{0}, \eta<\delta_{0}$, and $N(T) \leq \varepsilon$ for some positive constants $\delta_{0}$ and $\varepsilon$. Then it follows that

$$
N^{2}(T)+\int_{0}^{T}\left\|\left(\phi_{t}, \phi_{x}, \psi_{t}, \psi_{x}\right)(t)\right\|_{H^{2}}^{2} d t \leq C\left(N^{2}(0)+\delta_{0}^{2}\right),
$$

for $(\phi, \psi) \in X(0, T)$.

This lemma can be established by the following lemmas 4.3-4.5.

By the Sobolev embedding theorem, $H^{m+1} \hookrightarrow C^{m}, m \geq 0$. Thus if $N(T) \leq \varepsilon$, then

$$
\|(\phi, \psi)\|_{C^{2}} \leq C \varepsilon, \quad\left\|\psi_{t}\right\|_{C^{1}} \leq C \varepsilon .
$$

From these facts, we know that there are constants $0<c<d<+\infty$ such that $c>c_{1}$ and $d<d_{1}$, and $v \in[c, d]$, which verifies the conditions $\left.\left.H_{1}\right)-H_{4}\right)$.

Lemma 4.3. Suppose that the conditions in Lemma 4.2 are satisfied, $\delta \leq \delta_{0}, \eta<\delta_{0}$, and $N(T) \leq \varepsilon$ for some suitably small $\delta_{0}$ and $\varepsilon$. Then we have

$$
\begin{aligned}
\sup _{0 \leq t \leq T} & \left\|\left(\phi, \psi, \phi_{x}, \psi_{t}, \psi_{x}\right)(t)\right\|^{2}+\int_{0}^{t}\left\|\left(\bar{v}_{\xi}^{\frac{1}{2}} \psi, \psi_{x}, \phi_{x}, \psi_{t}\right)(\tau)\right\|^{2} d \tau \\
& \leq C\left(N^{2}(0)+\delta_{0}^{2}+\delta_{0} N^{2}(t)\right)+C\left(\delta_{0}+\varepsilon\right) \int_{0}^{t}\left\|\left(\phi_{x}, \psi_{x x}\right)(\tau)\right\|^{2} d \tau .
\end{aligned}
$$

Proof. We consider the equality

$$
\left(\phi+\mu \psi_{x}\right) L_{1}+A^{-1}\left(\mu \psi_{t}+\psi\right) L_{2}=\left(\phi+\mu \psi_{x}\right) F_{6}+A^{-1}\left(\psi+\mu \psi_{t}\right) R(x, t)
$$

with a positive constant $\mu$ such that $1<\mu=\frac{K+K_{1}}{2 K_{1}}<\frac{K}{K_{1}}$ and $K_{1}=\sup _{v \in[c . d]}\left|p_{E}^{\prime}(v)\right|$. The left-hand side of (4.10) can be reduced to

$$
\begin{aligned}
& {\left[\frac{1}{2} \phi^{2}+\frac{1}{2} A^{-1} \psi^{2}+\mu \phi \psi_{x}+\frac{\mu}{2} A^{-1} \psi_{t}^{2}+A^{-1} \psi \psi_{t}+\frac{\mu K}{2} A^{-1} \psi_{x}^{2}\right]_{t}} \\
& +\left[(\mu-1) A^{-1}-\frac{1}{2} \mu A_{t}^{-1}\right] \psi_{t}^{2}+\left[K A^{-1}-\frac{1}{2} \mu K A_{t}^{-1}-\mu\right] \psi_{x}^{2} \\
& \quad-\frac{1}{2} A_{t}^{-1} \psi^{2}-A_{t}^{-1} \psi \psi_{t}+K A_{x}^{-1} \psi \psi_{x}+K \mu A_{x}^{-1} \psi_{t} \psi_{x}+\{\cdots\}_{x}
\end{aligned}
$$

where $\{\cdots\}_{x}$ denotes the terms that disappear after integrations with respect to $x$.

It is easy to see that

$$
\begin{aligned}
& b_{1}\left(\psi^{2}+\psi_{t}^{2}\right) \leq\left[\frac{1}{2} A^{-1} \psi^{2}+\frac{\mu}{2} A^{-1} \psi_{t}^{2}+A^{-1} \psi \psi_{t}\right] \\
& \leq b_{2}\left(\psi^{2}+\psi_{t}^{2}\right), \\
& b_{3}\left(\phi^{2}+\psi_{x}^{2}\right) \leq\left[\frac{1}{2} \phi^{2}+\mu \phi \psi_{x}+\frac{\mu K}{2} A^{-1} \psi_{x}^{2}\right] \\
& \leq b_{4}\left(\phi^{2}+\psi_{x}^{2}\right), \\
& {\left[(\mu-1) A^{-1}-\frac{1}{2} \mu A_{t}^{-1}\right] \psi_{t}^{2} \geq b_{5} \psi_{t}^{2}, } \\
& {\left[K A^{-1}-\frac{1}{2} \mu K A_{t}^{-1}-\mu\right] \psi_{x}^{2} \geq b_{6} \psi_{x}^{2}, }
\end{aligned}
$$


for some positive constants $b_{i}(i=1, \ldots, 6)$.

We also note that

$$
-\frac{1}{2} A_{t}^{-1} \psi^{2} \geq b_{7} \bar{v}_{\xi} \psi^{2}
$$

for some positive constant $b_{7}$. Then by the Cauchy-Schwarz inequality, we have

$$
\begin{aligned}
\left|A_{t}^{-1} \psi \psi_{t}\right| & \leq \frac{1}{2} b_{7} \bar{v}_{\xi} \psi^{2}+C \eta \psi_{t}^{2}, \\
\left|K A_{x}^{-1} \psi \psi_{x}\right| & \leq \frac{1}{4} b_{7} \bar{v}_{\xi} \psi^{2}+C \eta \psi_{x}^{2}, \\
\left|K \mu A_{x}^{-1} \psi_{t} \psi_{x}\right| & \leq C \eta\left(\psi_{x}^{2}+\psi_{t}^{2}\right) .
\end{aligned}
$$

Integrating (4.10) over $[0, t] \times(-\infty,+\infty)$, taking $\eta$ suitably small, we arrive at

$$
\begin{aligned}
& \left\|\left(\phi, \psi, \psi_{t}, \psi_{x}\right)(t)\right\|^{2}+\int_{0}^{t}\left\|\left(\psi_{x}, \psi_{t}\right)(\tau)\right\|^{2} d \tau+\int_{0}^{t} \int_{-\infty}^{+\infty} \bar{v}_{\xi} \psi^{2} d x d \tau \\
& \leq C N^{2}(0)+C\left|\int_{0}^{t} \int_{-\infty}^{+\infty}\left[\left(\phi+\mu \psi_{x}\right) F_{6}+A^{-1}\left(\psi+\mu \psi_{t}\right) R(x, t)\right] d x d \tau\right| .
\end{aligned}
$$

We will estimate the right-hand side of (4.14) next.

At first, we claim that there exist positive constants $b_{8}$ and $b_{9}$ such that

$$
\int_{-\infty}^{+\infty}\left|\bar{v}(x, t)-v_{-} \| m(x, t)\right| d x \leq b_{8} \eta \delta \exp \left(-b_{9}(t+1)\right) .
$$

In fact, it follows from Lemma 2.3 that

$$
\begin{aligned}
\left|\bar{v}(\xi)-v_{-}\right| & \leq \int_{-\infty}^{\xi}\left|\bar{v}_{\xi}(y)\right| d y \leq C \eta \exp (C \xi), \quad \xi<0, \\
\left|\bar{v}-v_{-}\right| & \leq C \eta, \quad \xi \in \mathbf{R}^{1} .
\end{aligned}
$$

We may write $m(z)$ as

$$
m(\xi, t)=\frac{\delta}{\sqrt{2 d \pi}} \exp \left(-\frac{1}{2 d}\left(\frac{\xi+\left(\sigma-\lambda_{1}\right)(t+1)-\sigma}{\sqrt{t+1}}\right)^{2}\right) .
$$

We note that $\sigma-\lambda_{1}>0$. It is easy to verify (4.15) by dividing the integral interval into $\left(-\infty,-\frac{1}{2}\left(\sigma-\lambda_{1}\right)(t+1)-x_{0}+\sigma t\right),\left(-\frac{1}{2}\left(\sigma-\lambda_{1}\right)(t+1)-x_{0}+\sigma t,-x_{0}+\sigma t\right)$, and $\left(-x_{0}+\sigma t,+\infty\right)$, and then using $(4.16)_{1}$ and $(4.16)_{2}$ on the different intervals. Similar estimates hold when we use the derivatives of $m$ and $h(\bar{v})-h\left(v_{-}\right)$(or $\bar{v}_{t}$ ) instead of $m$ and $\bar{v}-v_{-}$in (4.15), respectively, for some $C^{1}$ function $h$.

Next, we make some analysis on the term $R(x, t)$. By (4.15), we have

$$
\int_{0}^{t} \int_{-\infty}^{+\infty}\left(\left|G_{1}\right|+\left|I_{3}\right|+\left|I_{4}\right|\right) d x d \tau \leq C \delta \eta
$$

Since $\eta, \delta$, and $N(t)$ are small, it is easy to check that

$$
I_{5}=\left[F_{9}+F_{10}\right] \text {, }
$$

where $F_{9}=O(1)\left(m+m_{x}\right) \bar{v}_{t}, F_{10}=O(1)\left(\phi_{x}+\psi_{t}\right) \bar{v}_{t}$, and then

$$
\int_{0}^{t} \int_{-\infty}^{+\infty}\left|F_{9}\right| d x d \tau \leq C \delta \eta
$$


Hence, we can divide $R(x, t)$ as follows:

$$
R(x, t)=E(x, t)+H(x, t)-I_{1}-I_{2}-I_{6}-I_{7},
$$

with

$$
\begin{gathered}
\int_{0}^{t} \int_{-\infty}^{+\infty}|E(x, t)| d x d \tau \leq C \delta \eta \\
H(x, t)=O(1)(t+1)^{-1}\left[(z m)_{x}+(z m)_{x x}+(z m)_{t x}\right]+F_{10} .
\end{gathered}
$$

We may estimate the typical terms with $E(x, t)$ or $H(x, t)$ involved as

$$
\begin{gathered}
\int_{0}^{t} \int_{-\infty}^{+\infty}\left|A^{-1}\left(\psi+\mu \psi_{x}\right)\right| E(x, t) \mid d x d \tau \leq C N(t) \delta \eta \\
\leq C \delta^{2}+\eta^{2} N^{2}(t), \\
\left|\int_{0}^{t} \int_{-\infty}^{+\infty} A^{-1} \mu \psi_{t} H(x, t) d x d \tau\right| \\
\leq \varepsilon_{1} \int_{0}^{t} \int_{-\infty}^{+\infty} \psi_{t}^{2} d x d \tau+C\left(\varepsilon_{1}\right) \int_{0}^{t} \int_{-\infty}^{+\infty} H^{2} d x d \tau \\
\leq\left(\varepsilon_{1}+\eta C\left(\varepsilon_{1}\right)\right) \int_{0}^{t}\left\|\left(\psi_{t}, \phi_{x}\right)(\tau)\right\|^{2} d x d \tau+C\left(\varepsilon_{1}\right) \delta^{2}, \\
\left|\int_{0}^{t} \int_{-\infty}^{+\infty} A^{-1} \psi H(x, t) d x d \tau\right| \\
=\left|\int_{0}^{t} \int_{-\infty}^{+\infty}\left[A_{x}^{-1} \psi+A^{-1} \psi_{x}\right] H_{1}(x, t) d x d \tau\right|+\left|\int_{0}^{t} \int_{-\infty}^{+\infty} A^{-1} \psi F_{10} d x d \tau\right| \\
\leq\left(\varepsilon_{2}+C \eta\right) \int_{0}^{t} \int_{-\infty}^{+\infty}\left[\bar{v}_{\xi} \psi^{2}+\psi_{x}^{2}+\psi_{t}^{2}+\phi_{x}^{2}\right] d x d \tau+C\left(\varepsilon_{2}\right) \delta^{2},
\end{gathered}
$$

where $\varepsilon_{1}, \varepsilon_{2}$ are arbitrary positive numbers and

$$
H_{1}(x, t)=O(1)(t+1)^{-1}\left[(z m)+(z m)_{x}+(z m)_{t}\right]
$$

We also note that

$$
\begin{aligned}
& \left|\int_{0}^{t} \int_{-\infty}^{+\infty}\left(\phi+\mu \psi_{t}\right) F_{6} d x d \tau\right| \\
& \quad \leq \varepsilon_{3} \int_{0}^{t}\left\|\left(\phi_{x}, \psi_{t}\right)(\tau)\right\|^{2} d \tau+C\left(\varepsilon_{3}\right) \delta^{2}
\end{aligned}
$$

for $\varepsilon_{3}>0$ suitably small.

We now turn to the terms with $I_{1}, I_{2}, I_{6}$, and $I_{7}$. Due to the smallness of $\eta$ and $\delta$, we have, with the help of Taylor's theorem, that

$$
F_{1}=O(1)\left[(t+1)^{-\frac{1}{2}}\left(m+m_{z}\right)+F_{7}+\left(\phi_{x}+\psi_{t}\right)\right]
$$


The method used in (4.22)-(4.25) and the Cauchy-Schwarz inequality give

$$
\begin{aligned}
& \left|\int_{0}^{t} \int_{-\infty}^{+\infty} A^{-1}\left(\psi+\mu \psi_{t}\right)\left(I_{1}+I_{2}\right) d x d \tau\right| \\
& \quad \leq C\left(\varepsilon_{4}\right) \delta^{2}+\varepsilon_{4} \int_{0}^{t} \int_{-\infty}^{+\infty}\left(\bar{v}_{\xi} \psi^{2}+\phi_{x}^{2}+\psi_{x}^{2}+\psi_{t}^{2}\right) d x d \tau
\end{aligned}
$$

with a small positive number $\varepsilon_{4}$.

For the terms with $I_{7}$, we note that

$$
I_{7}=-F_{2}\left(\phi_{x t}\right)=-F_{2} \psi_{x x}-F_{2} F_{6 x}
$$

Similar to (4.23)-(4.24), it is easy to verify that

$$
\begin{aligned}
& \left|\int_{0}^{t} \int_{-\infty}^{+\infty} A^{-1}\left(\psi+\mu \psi_{t}\right) F_{2} F_{6 x} d x d \tau\right| \\
& \quad \leq C\left(\varepsilon_{5}\right) \delta^{2}+\varepsilon_{5} \int_{0}^{t} \int_{-\infty}^{+\infty}\left(\bar{v}_{\xi} \psi^{2}+\psi_{t}^{2}\right) d x d \tau
\end{aligned}
$$

for a positive number $\varepsilon_{5}$. We also can show that

$$
\begin{aligned}
\left|\int_{0}^{t} \int_{-\infty}^{+\infty} A^{-1} F_{2}\left(\psi+\mu \psi_{t}\right) \psi_{x x} d x d \tau\right| \\
=\left|\int_{0}^{t} \int_{-\infty}^{+\infty}\left[\left(A^{-1} F_{2}\right)_{x} \psi+A^{-1} F_{2} \psi_{x}\right] \psi_{x} d x d \tau\right| \\
\quad+C \eta \int_{0}^{t}\left\|\left(\psi_{t}, \psi_{x x}\right)(\tau)\right\|^{2} d \tau \\
\quad \leq C \eta \int_{0}^{t} \int_{-\infty}^{+\infty} \bar{v}_{\xi} \psi^{2} d x d \tau+C \eta \int_{0}^{t}\left\|\left(\psi_{x}, \psi_{t}, \psi_{x x}\right)(\tau)\right\|^{2} d \tau
\end{aligned}
$$

The estimates for the terms with $I_{7}$ can then be obtained by (4.27)-(4.28).

For the terms with $I_{6}$ we deduce that

$$
I_{6}=O(1)\left[(t+1)^{-\frac{1}{2}}\left(m+m_{z}\right)+F_{7}+\phi_{x}+\psi_{t}\right]\left[\psi_{x x}+F_{6 x}\right]
$$

All of the terms involved can be treated by the similar approach mentioned above except

$$
(t+1)^{-\frac{1}{2}}\left(m+m_{z}\right) \psi_{x x}=(t+1)^{-\frac{1}{2}} m \psi_{x x}+m_{x} \psi_{x x}
$$

In the following we only deal with the term $(t+1)^{-\frac{1}{2}} m \psi_{x x}$, since $m_{x} \psi_{x x}$ can be treated similarly. Due to the properties of $m$, we have

$$
\left|\int_{0}^{t} \int_{-\infty}^{+\infty} A^{-1} \mu \psi_{t}(t+1)^{-\frac{1}{2}} m \psi_{x x} d x d \tau\right| \leq C \delta \int_{0}^{t}\left\|\left(\psi_{t}, \psi_{x x}\right)(\tau)\right\|^{2} d \tau
$$


and

$$
\begin{aligned}
\left|\int_{0}^{t} \int_{-\infty}^{+\infty} A^{-1} \psi(t+1)^{-\frac{1}{2}} m \psi_{x x} d x d \tau\right| \\
=\left|\int_{0}^{t} \int_{-\infty}^{+\infty}\left[A_{x}^{-1} \psi+A^{-1} \psi_{x}\right](t+1)^{-\frac{1}{2}} m \psi_{x}+A^{-1}(t+1)^{-\frac{1}{2}} m_{x} \psi \psi_{x} d x d \tau\right| \\
\leq C \delta \int_{0}^{t} \int_{-\infty}^{+\infty}\left[\bar{v}_{\xi} \psi^{2}+\psi_{x}^{2}\right] d x d \tau \\
\quad+C\left|\int_{0}^{t} \int_{-\infty}^{+\infty}\left[\left(A^{-1}(t+1)^{-\frac{1}{2}} m_{x}\right)_{x} \psi^{2}\right] d x d \tau\right| \\
\leq C \delta \int_{0}^{t} \int_{-\infty}^{+\infty}\left[\bar{v}_{\xi} \psi^{2}+\psi_{x}^{2}\right] d x d \tau \\
\left.\quad+C \mid \int_{0}^{t} \int_{-\infty}^{+\infty}(t+1)^{-\frac{3}{2}}\left(m+z^{2} m\right) \psi^{2}\right] d x d \tau \mid \\
\leq C \delta \int_{0}^{t} \int_{-\infty}^{+\infty}\left[\bar{v}_{\xi} \psi^{2}+\psi_{x}^{2}\right] d x d \tau+C \delta \int_{0}^{t}(t+1)^{-\frac{3}{2}}\|\psi(\tau)\|^{2} d \tau \\
\leq C \delta \int_{0}^{t} \int_{-\infty}^{+\infty}\left(\bar{v}_{\xi} \psi^{2}+\psi_{x}^{2}\right) d x d \tau+C \delta N^{2}(t) .
\end{aligned}
$$

The combination of (4.14)-(4.30) with the $\varepsilon_{i}(i=1, \ldots, 5), \delta, \eta$, and $\varepsilon$ suitably small yields that

$$
\begin{aligned}
& \sup _{0 \leq t \leq T}\left\|\left(\phi, \psi, \psi_{t}, \psi_{x}\right)(t)\right\|^{2}+\int_{0}^{t}\left\|\left(\psi_{x}, \psi_{t}\right)(\tau)\right\|^{2} d \tau+\int_{0}^{t} \int_{-\infty}^{+\infty} \bar{v}_{\xi} \psi^{2} d x d \tau \\
& \leq C\left(N^{2}(0)+\delta^{2}+\delta_{0} N^{2}(t)\right)+C\left(\delta+\varepsilon+\varepsilon_{6}\right) \int_{0}^{t}\left\|\left(\phi_{x}, \psi_{x x}\right)(\tau)\right\|^{2} d \tau
\end{aligned}
$$

for some small positive number $\varepsilon_{6}$.

To bound $\phi_{x}$, we investigate the following relation:

$$
\left(K \phi_{x}-\psi_{t}\right) \partial_{x} L_{1}-\phi_{x} L_{2}=\left(K \phi_{x}-\psi_{t}\right) F_{6 x}-\phi_{x} R(x, t) .
$$

The left-hand side of (4.32) can be reduced into

$$
\left[\frac{1}{2} K \phi_{x}^{2}-\psi_{t} \phi_{x}-\frac{1}{2} \psi_{x}^{2}\right]_{t}-\phi_{x} \psi_{t}+A \phi_{x}^{2}+\{\cdots\}_{x}
$$

We know that $A>a_{1}>0$ from $H_{1}$. Integrating (4.32) over $[0, t] \times(-\infty,+\infty)$, and using Young's inequality, (4.31), and a same argument as used in the proof of (4.31) to deal with the terms involving $R(x, t)$, we show that

$$
\begin{aligned}
& \left\|\phi_{x}(t)\right\|^{2}+\int_{0}^{t}\left\|\phi_{x}(\tau)\right\|^{2} d \tau \\
& \quad \leq C\left(N^{2}(0)+\delta_{0}^{2}+\delta_{0} N^{2}(t)\right)+C\left(\varepsilon+\delta_{0}+\varepsilon_{6}\right) \int_{0}^{t}\left\|\left(\phi_{x}, \psi_{x x}\right)(\tau)\right\|^{2} d \tau .
\end{aligned}
$$

We choose $\varepsilon_{6}, \varepsilon$, and $\delta_{0}$ suitably small such that $C\left(\varepsilon+\delta_{0}+\varepsilon_{6}\right) \leq \frac{1}{2}$. Then we obtain (4.9) by combining (4.31) and (4.34). 
We turn to the higher-order estimates next. To this end, instead of (4.10) and (4.32), we study the following equations:

$$
\begin{aligned}
& \left(\phi_{x}+\mu \psi_{x x}\right) \partial_{x} L_{1}+A^{-1}\left(\mu \psi_{x t}+\psi_{x}\right) \partial_{x} L_{2} \\
& \quad=\left(\phi_{x}+\mu \psi_{x x}\right) F_{6 x}+A^{-1}\left(\psi_{x}+\mu \psi_{t x}\right) R(x, t)_{x}
\end{aligned}
$$

and

$$
\left(K \phi_{x x}-\psi_{t x}\right) \partial_{x x} L_{1}-\phi_{x x} \partial_{x} L_{2}=\left(K \phi_{x x}-\psi_{t x}\right) F_{6 x x}-\phi_{x x} R(x, t)_{x}
$$

Repeating the procedure as in the proof of Lemma 4.3, we can prove

Lemma 4.4. Suppose that the conditions in Lemma 4.2 are satisfied, $\delta \leq \delta_{0}, \eta<\delta_{0}$, and $N(T) \leq \varepsilon$ for some suitably small $\delta_{0}$ and $\varepsilon$. Then we have

$$
\begin{aligned}
& \sup _{0 \leq t \leq T}\left\|\left(\phi_{x}, \psi_{x}, \phi_{x x}, \psi_{x x}, \psi_{t x}\right)(t)\right\|^{2}+\int_{0}^{t}\left\|\left(\psi_{x x}, \phi_{x x}, \psi_{t x}\right)(\tau)\right\|^{2} d \tau \\
& \quad \leq C\left(N^{2}(0)+\delta_{0}^{2}+\delta_{0} N^{2}(t)\right) .
\end{aligned}
$$

Now we prove the third-order estimates. Similar to [3] and [13], we have

Lemma 4.5. Suppose the conditions in Lemma 4.2 are satisfied, $\delta \leq \delta_{0}, \eta \leq \delta_{0}$, and $N(T) \leq \varepsilon$ for some suitably small $\delta_{0}$ and $\varepsilon$. Then we have

$$
\begin{aligned}
& \sup _{0 \leq t \leq T}\left\|\left(\phi_{x x x}, \psi_{x x x}, \psi_{t x x}\right)(t)\right\|^{2}+\int_{0}^{t}\left\|\left(\psi_{x x x}, \phi_{x x x}, \psi_{t x x}\right)(\tau)\right\|^{2} d \tau \\
& \leq C\left(N^{2}(0)+\delta_{0}^{2}+\delta_{0} N^{2}(t)\right) .
\end{aligned}
$$

Proof. We make use of the following equality:

$$
\begin{aligned}
\left(\phi_{x x}\right. & \left.+\mu \psi_{x x x}\right) \partial_{x x} L_{1}+A^{-1}\left(\mu \psi_{t x x}+\psi_{x x}\right) \partial_{x x} L_{2} \\
& =\left(\phi_{x x}+\mu \psi_{x x x}\right) F_{6 x x}+A^{-1}\left(\psi_{x x}+\mu \psi_{t x x}\right) R(x, t)_{x x}
\end{aligned}
$$

Similar to the proof of Lemma 4.3, one can easily get

$$
\begin{aligned}
\|\left(\phi_{x x},\right. & \left.\psi_{x x}, \psi_{t x x}, \psi_{x x x}\right)(t)\left\|^{2}+\int_{0}^{t}\right\|\left(\psi_{x x x}, \psi_{t x x}\right)(\tau) \|^{2} d \tau \\
\leq & C\left(N^{2}(0)+\delta_{0}^{2}+\delta_{0} N^{2}(t)\right) \\
& +C\left|\int_{0}^{t} \int_{-\infty}^{+\infty}\left[\left(\phi_{x x}+\mu \psi_{x x x}\right) F_{6 x x}+A^{-1}\left(\psi_{x x}+\mu \psi_{t x x}\right) R_{x x}(x, t)\right] d x d \tau\right| .
\end{aligned}
$$

To estimate the right-hand side of (4.40), we can employ a similar method as used before except for the terms with $I_{6}$ (let us denote them by $J$ ) which can be treated as follows.

It is easy to see that

$$
I_{6}=O(1)\left[(t+1)^{-\frac{1}{2}}\left(m+m_{z}\right)+F_{7}+\dot{\phi}_{x}+\psi_{t}\right]\left[\dot{\psi}_{x x}+F_{6 x}\right] .
$$

In view of the smallness of $\delta, \eta$, and $\varepsilon$, a similar argument as used before gives the estimates for all the terms in $J$ but

$$
A^{-1}\left[\left(\phi_{x}+\psi_{t}\right) \psi_{x x}\right]_{x x} \psi_{t x x}
$$


for which we have

$$
\begin{aligned}
\left|\int_{0}^{t} \int_{-\infty}^{+\infty} A^{-1} \psi_{t x x}\left[\left(\phi_{x}+\psi_{t}\right) \psi_{x x}\right]_{x x} d x d \tau\right| \\
=\left|\int_{0}^{t} \int_{-\infty}^{+\infty}\left[A_{x}^{-1} \psi_{t x x}+A^{-1} \psi_{t x x x}\right]\left[\left(\phi_{x}+\psi_{t}\right) \psi_{x x}\right]_{x} d x d \tau\right| \\
\leq C\left(\varepsilon+\delta_{0}\right) \int_{0}^{t}\left\|\left(\psi_{x x x}, \psi_{t x x}, \phi_{x x x}\right)(\tau)\right\|^{2} d \tau+C\left(\delta_{0}^{2}+N^{2}(0)+\delta_{0} N^{2}(t)\right) \\
\quad+C\left|\int_{0}^{t} \int_{-\infty}^{+\infty} A^{-1}\left[\left(\phi_{x}+\psi_{t}\right) \psi_{x x x} \psi_{t x x x}\right] d x d \tau\right| \\
\leq C\left(\varepsilon+\delta_{0}\right) \int_{0}^{t}\left\|\left(\psi_{t x x}, \psi_{x x x}, \phi_{x x x}\right)(\tau)\right\|^{2} d \tau \\
\quad+C\left(\delta_{0}^{2}+N^{2}(0)+\delta_{0} N^{2}(t)\right)+C\left(\delta_{0}+\varepsilon\right)\left\|\psi_{x x x}(t)\right\|^{2}
\end{aligned}
$$

Thus, we arrive at

$$
\begin{aligned}
\sup _{0 \leq t \leq T} & \left\|\left(\phi_{x x}, \psi_{x x}, \psi_{x x x}, \psi_{t x x}\right)(t)\right\|^{2}+\int_{0}^{t}\left\|\left(\psi_{x x x}, \psi_{t x x}\right)(\tau)\right\|^{2} d \tau \\
\leq & C\left(N^{2}(0)+\delta_{0}^{2}+\delta_{0} N^{2}(t)\right)+C\left(\delta_{0}+\varepsilon\right) \int_{0}^{t}\left\|\phi_{x x x}(\tau)\right\|^{2} d \tau
\end{aligned}
$$

For the estimate of $\phi_{x x x}$, we study the following equality:

$$
\left(K \phi_{x x x}-\psi_{t x x}\right) \partial_{x x x} L_{1}-\phi_{x x x} \partial_{x x} L_{2}=\left(K \phi_{x x x}-\psi_{t x x}\right) F_{6 x x x}-\phi_{x x x} R(x, t)_{x x}
$$

in the same manner as used in Lemma 4.3 to bound $\phi_{x}$. Keeping in mind the results in Lemmas 4.3 and 4.4 , by using the same argument as used in the proof of (4.42) to deal with the terms with $R(x, t)$ and noting the expression

$$
I_{6}=O(1)\left[(t+1)^{-\frac{1}{2}}\left(m+m_{z}\right)+F_{7}+\phi_{x}+\psi_{t}\right] \phi_{t x},
$$

we are able to show that

$$
\left\|\phi_{x x x}(t)\right\|^{2}+\int_{0}^{t}\left\|\phi_{x x x}(\tau)\right\|^{2} d \tau \leq C\left(N^{2}(0)+\delta_{0}^{2}+\delta_{0} N^{2}(t)\right),
$$

in view of the estimate (4.42). Then, (4.38) follows from (4.42) and (4.44).

The estimates for $\phi_{t}, \phi_{x t}, \phi_{t t}$, and $\phi_{t x x}$ can be derived from the above lemmas and the relation (4.4).

We now prove Lemma 4.2. In view of Lemmas 4.3-4.5, we have for any $t \in[0, T]$ that

$$
N^{2}(t)+\int_{0}^{t}\left\|\left(\phi_{x}, \psi_{x}, \psi_{t}, \phi_{t}\right)(\tau)\right\|_{H^{2}}^{2} d \tau \leq C\left(N(0)^{2}+\delta_{0}^{2}+\delta_{0} N^{2}(t)\right) .
$$

By choosing $\delta_{0}$ small such that $C \delta_{0} \leq \frac{1}{2}$, we have proved Lemma 4.2.

Let us turn to the problem (4.4)-(4.5) and (4.8) next. Since the local (in time) existence and uniqueness of the solution for the initial value problem (4.4)-(4.5) and (4.8) can be obtained by standard procedures in view of the a priori estimates in Lemma 4.2, it follows from Lemma 4.2 and a standard continuity argument (cf. [3], [4], [5], $[6],[11]$, and [13]) that the problem (4.4)-(4.5) and (4.8) has a unique global (in time) 
solution $(\phi, \psi) \in X(0,+\infty)$, satisfying the estimates cited in Lemma 4.2 for any $t \geq 0$, namely,

$$
\|(\phi, \psi)(t)\|_{H^{3}}^{2}+\left\|\psi_{t}(t)\right\|_{H^{2}}^{2}+\int_{0}^{t}\left\|\left(\phi_{x}, \psi_{x}, \psi_{t}, \phi_{t}\right)(\tau)\right\|_{H^{2}}^{2} d \tau \leq C\left(N(0)^{2}+\delta_{0}^{2}\right) .
$$

Due to (4.45), it is known that

$$
\int_{0}^{t}\left|\frac{d}{d t} \int_{-\infty}^{+\infty}\left\|\phi_{x}\right\|_{H^{2}}^{2}(x, \tau) d x\right| d \tau \leq C .
$$

Thus

$$
\int_{0}^{+\infty}\left(\left\|\phi_{x}(t)\right\|_{H^{2}}^{2}+\left(\left|\frac{d}{d t}\left\|\phi_{x}(t)\right\|_{H^{2}}^{2}\right|\right) d t<+\infty\right.
$$

It follows then that

$$
\lim _{t \rightarrow+\infty}\left\|\phi_{x}(t)\right\|_{H^{2}}^{2}=0
$$

Similarly, it can be proved that

$$
\lim _{t \rightarrow+\infty}\left\|\left(\psi_{x}, \psi_{t}\right)\right\|_{H^{2}}=0
$$

So Sobolev's inequality implies that

$$
\begin{aligned}
\lim _{t \rightarrow+\infty} & \sup _{x \in \mathbf{R}}|(\phi, \psi)(x, t)| \\
& \leq \lim _{t \rightarrow+\infty}\left(\|(\phi, \psi)\| \|\left(\phi_{x}, \psi_{x} \|\right)^{\frac{1}{2}}\right. \\
& \leq C \lim _{t \rightarrow+\infty}\left\|\left(\phi_{x}, \psi_{x}\right)(t)\right\|^{\frac{1}{2}} \\
& =0 .
\end{aligned}
$$

This completes the proof of Theorem 4.1. Then the proof of Theorem 1 is completed.

Acknowledgment. The authors are grateful to Dr. Luo Tao for stimulating discussions. Special thanks go to the referee for the comments in improving the presentation of this paper.

\section{REFERENCES}

[1] T. Chang and L. Hsiao, The Riemann problem and interaction of waves in gas dynamics, Pitman Monographs and Surveys in Pure and Appl. Math. 41, Longman Sci. and Tech., John Wiley and Sons, New York, 1989

[2] G. H. Hardy, J. E. Littlewood, and G. Polya, Inequalities, Cambridge Univ. Press, 1934

[3] L. Hsiao and T. Luo, Stability of travelling wave solutions for a rate-type viscoelastic system, Advances in Nonlinear Partial Differential Equations and Related Areas, World Sci. Publishing, River Edge, NJ, 1998

[4] L. Hsiao and R. H. Pan, Nonlinear stability of rarefaction waves for a rate-type viscoelastic system, Chinese Ann. Math. 20 B:2, 223-232 (1999)

[5] L. Hsiao and R. H. Pan, Nonlinear stability of two-mode shock profiles for a rate-type viscoelastic system with relaxation, Chinese Ann. Math. 20 B:4, 479-488 (1999)

[6] S. Kawashima and A. Matsumura, Asymptotic stability to travelling wave solutions of systems for one-dimensional gas motion, Comm. Math. Phys. 101, 97-127 (1985)

[7] R. J. LeVeque, H. C. Yee, P. Roe, and B. V. Leer, Model systems for reacting flow, Final Report, NASA-Ames Univ. Consortium NCA2-185 (1988)

[8] T. P. Liu, Hyperbolic conservation laws with relaxation, Comm. Math. Phys. 108, 153-175 (1987) 
[9] T. P. Liu, Nonlinear stability of shock waves for viscous conservation laws, Memoirs of Amer. Math. Soc. 328 (1985)

[10] T. Luo, Asymptotic stability of planar rarefaction waves for the relaxation approximations of conservation laws in several dimensions, J. Differential Equations 133, 255-279 (1997)

[11] T. Luo and D. Serre, Linear stability of shock profiles for a rate-type viscoelastic system with relaxation, Quart. Appl. Math. 56, 569-586 (1998)

[12] T. Luo and Z. P. Xin, Asymptotic stability of planar shock profiles for the relaxation approximations of conservation laws in several dimensions, J. Differential Equations 139, 365-408 (1997)

[13] R. H. Pan, The nonlinear stability of traveling wave solutions for a reacting flow model with source term, Acta Math. Sci. 19, 26-36 (1999)

[14] A. Szepessy and Z. P. Xin, Nonlinear stability of viscous shock waves, Arch. Rational Mech. Anal. 122, 53-103 (1993)

[15] W.-A. Yong, Existence and asymptotic stability of traveling wave solutions of a model system for reacting flow, Nonlinear Analysis 26, 1791-1809 (1996) 